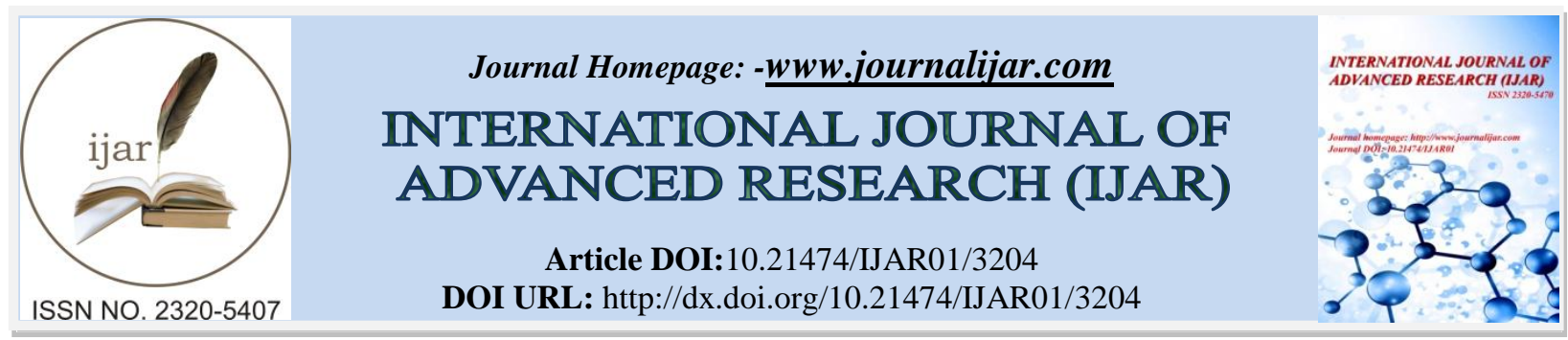

RESEARCH ARTICLE

\title{
COMPARISON OF EARLY RESULTS BETWEEN ON-PUMP AND OFF-PUMP CABG IN PATIENTS WITH PREOPERATIVE MILD TO MODERATE RENAL IMPAIRMENT.
}

Ali Emad Ashraf Abdel Aziz, Tamer Elbanna, Mostafa Mansy and Amr Alprince.

M.D. Cardiothoracic surgery, National Heart Institute, Egypt.

\section{Manuscript Info}

Manuscript History

Received: 20 Decmber 2016

Final Accepted: 04 January 2017

Published: February 2017

Key words:-

CABG, Cardiac surgery, OFF pump, Renal impairment.

\section{Abstract}

Objective: to compare the early results of isolated $\mathrm{CABG}$, using onpump and off-pump, in patients with preoperative mild to moderate elevation of serum creatinine (1.6 to $2.5 \mathrm{mg} / \mathrm{dl}$ ) level whom do not require maintenance dialysis to support renal function.

Methods: This prospective non-randomized study included 60 patients who complained of symptoms of Coronary Artery Disease and subsequently underwent myocardial revascularization at National Heart Institute of Egypt, from July 2013 till May 2014.

Results: no statistically significant difference between the two groups except for:

- The Hemoglobin, which is lower in on-pump group due to hemodilution effect of CPB

- The troponin, as we discussed before, the 6 patients who had recent infarction we preferred to do them on-pump.

- Kidney function tests

Conclusions: In general, there is no single accurate investigation that can surely predict renal function outcome after cardiac surgery. - $\square$ General Measures to prevent renal dysfunction after Cardiac Surgery should be utilized in all patients with special attention to those with preoperative renal dysfunction. $\bullet \square$ Regarding postoperative renal dysfunction and need for dialysis, results were in favor of the off-pump technique.

Copy Right, IJAR, 2017,. All rights reserved.

\section{Introduction:-}

The development of cardiac surgery in the last 30 years is directly related to the improvement of the techniques of Cardio-Pulmonary Bypass (CPB). However, CPB utilization constitutes one of the primary causes of perioperative complications.[1] In an attempt to avoid the above-mentioned complications, recently, there was a renewal interest in the performance of Coronary Artery Bypass Grafting (CABG) without using CPB, (off-pump).[2] Despite the improvements in surgical techniques, CPB circuit and postoperative patient care, renal dysfunction is still a major complication in patients undergoing cardiac surgery with CPB. It has been reported that acute renal failure requiring dialysis develops in $2-7 \%$ of cardiac surgery patients and is strongly associated with postoperative morbidity and mortality.[3] Although, the cause is multi-factorial and depends on the patient's clinical status, age, type I diabetes, recent exposure to nephrotoxic drugs, etc., yet the CPB-related events, such as hypotension, hypoperfusion, loss of pulsatility, hemolysis and release of proinflammatory substances, may contribute significantly to this condition.[4] In addition the duration of CPB has a considerable negative influence on alterations of kidney function integrity.[5] 
Although multiple risk factors have been entertained, the most consistent preoperative risk factor for developing renal insufficiency is preexisting renal insufficiency.[6] This is not surprising, because the incidence of postoperative acute renal failure in patients with previously normal renal function is less than 1\%.[7] Thus an elevated preoperative serum creatinine concentration $(1.5 \mathrm{mg} / \mathrm{dl})$ is an important independent risk factor for mortality and morbidity rates after cardiac surgery suggesting that mild elevations in serum creatinine concentrations are a red flag for bad outcome both before and after cardiac surgery.[8] Mild to moderate elevation of creatinine ( 1.6 to $2.5 \mathrm{mg} / \mathrm{dl}$ ) adds moderate risk, but preoperative values higher than $2.5 \mathrm{mg} / \mathrm{dl}$ are associated with markedly increased risk of postoperative dialysis and in-hospital mortality.[9] At least some of the factors that precipitate renal dysfunction after cardiac surgery are relatively well known. Haemodynamic changes probably play a major role, specifically vasoconstriction of the renal arterioles and the redistribution of blood flow within the kidneys, which leave parts of the renal parenchyma underperfused and thus hypoxic. Prolongation of this vasoconstricted state leads to permanent cellular damage; shorter periods result in transient dysfunction. Among the well known causes for renal vasoconstriction that are frequent in cardiac surgery are hypovolaemia, dehydration, and many other alterations of systemic haemodynamics. There are also hormonal changes that may adversely affect renal function, such as increased vasopressin, catecholamines, aldosterone, angiotensin, and decreased atrial natriuretic factor and nitric oxide.[10] In an attempt to reduce the incidence of this complication, several therapeutic strategies, such as pulsatile perfusion, hemofiltration, natriuretic hormone, have been investigated and used. Nevertheless, because there has been a trend in operating on higher risk patients with preoperative comorbidities, the development of this complication is still an important and challenging problem.[4] Advances in surgical instruments for the stabilization and visualization of an optimal surgical field have facilitated CABG without CPB.[4] Once the safety of off-pump coronary procedures had been established, the use of beating heart surgery has focused on improving operative outcome by eliminating the morbidity of cardiopulmonary bypass. Operative mortality rate, postoperative length of stay, and perioperative blood usage were improved by the use of off-pump CABG when compared with on-pump CABG.[11] The avoidance of CPB use may also provide beneficial effects on perioperative renal function by preserving pulsatility, and by preventing the adverse side effects of CPB.[4]

\section{Patients and Methods:-}

This prospective non-randomized study included 60 patients who complained of symptoms of Coronary Artery Disease and subsequently underwent myocardial revascularization at National Heart Institute of Egypt, from July 2013 till May 2014.

\section{Inclusion Criteria:-}

Any patient submitted to isolated elective $\mathrm{CABG}$ within the mentioned time interval with: A) Preoperative mild to moderate elevation of serum creatinine levels between 1.6 to $2.5 \mathrm{mg} / \mathrm{dl}$. Level who do not require maintenance dialysis to support renal function. AND B): 1- Multi-vessel coronary artery disease. 2- Patients undergoing isolated on-pump (conventional) surgery. 3- Patients undergoing isolated off-pump (Beating Heart) surgery. 4- Controlled risk factors e.g.: hypertension, diabetes mellitus, hyperlipidemia, and smoking.

\section{Exclusion Criteria:-}

1- Patients with normal serum creatinine levels. 2- Patients with single-vessel disease. 3- Patients undergoing emergency surgery. 4- Left ventricular ejection fraction $<30 \%$. 5- Patients planned for OPCAB and reverted to on pump due to heamodynamic instability. 6- Patients for redo CABG.

\section{Aim of the work:-}

In this study we compared the early results of isolated CABG, using on-pump and off-pump, in patients with preoperative mild to moderate elevation of serum creatinine (1.6 to $2.5 \mathrm{mg} / \mathrm{dl}$ ) level whom do not require maintenance dialysis to support renal function. We examined if off-pump coronary revascularization offers a superior renal protection when compared with conventional coronary revascularization with cardiopulmonary bypass.

\section{Methodology:-}

Patients were submitted for either group according to the surgeon's preference as follows: $\bullet \square$ Group A patients (the on-pump group): included those who underwent conventional myocardial revascularization by means of LIMA on LAD grafts plus additional SVGs. $\square$ Group B patients (the offpump group): included those who underwent off- 
pump myocardial revascularization by means of LIMA on LAD grafts plus additional SVGs. The 2 groups were similar with respect to age, sex and preoperativevariables.

\section{Statistical analysis:-}

Data was analyzed by Microsoft Office 2003 (excel) and Statistical Package for Social Science (SPSS) version 16. Parametric data was expressed as mean, SD, and non parametric data was expressed as number and percentage of the total. ${ }^{*} \mathrm{P}$ value $\leq 0.05$ is considered significant.

\section{Results:-}

Table 1:- showing demographic data among studied groups

\begin{tabular}{|l|l|l|l|l|l|}
\hline \multirow{2}{*}{ Demographic Data } & On - Pump & Of - Pump & \multirow{2}{*}{ P value } \\
\cline { 2 - 6 } & & & & & \\
\hline Age $_{\text {years }}$ & 73.40 & 6.38 & 72.87 & 9.62 & $\mathbf{0 . 8 0 1}$ \\
\hline $\mathbf{W t}_{\mathbf{k g}}$ & 83.13 & 12.68 & 79.47 & 8.92 & $\mathbf{0 . 2 0 1}$ \\
\hline
\end{tabular}

Table 2:- showing Gender distribution among studied groups.

\begin{tabular}{|l|l|l|l|l|}
\hline \multirow{2}{*}{ Gender } & On - Pump & Of - Pump \\
\cline { 2 - 5 } & Number & $\%$ & Number & $\%$ \\
\hline Females & 5 & $\mathbf{1 6 . 6 7}$ & 7 & $\mathbf{2 3 . 2 3}$ \\
\hline Males & 25 & $\mathbf{8 3 . 3 3}$ & 23 & $\mathbf{7 6 . 6 7}$ \\
\hline
\end{tabular}

Table 3:- showing risk factors among studied groups.

\begin{tabular}{|l|l|l|l|l|}
\hline \multirow{2}{*}{ Smoking } & On - Pump & Off - Pump \\
\cline { 2 - 5 } & Number & $\%$ & Number & $\%$ \\
\hline Hypertension & 8 & $\mathbf{2 6 . 6 7}$ & 9 & $\mathbf{3 0 . 0 0}$ \\
\hline D.M. & 27 & $\mathbf{9 0 . 0 0}$ & 24 & $\mathbf{8 0 . 0 0}$ \\
\hline Dyslipidemia & 16 & $\mathbf{5 3 . 3 3}$ & 14 & $\mathbf{4 6 . 6 7}$ \\
\hline Previous MI & 24 & $\mathbf{8 0 . 0 0}$ & 24 & $\mathbf{8 0 . 0 0}$ \\
\hline
\end{tabular}

Table 4:- showing previous MI among studied groups.

\begin{tabular}{|l|l|l|l|l|}
\hline \multirow{2}{*}{ Gender } & On - Pump & Of - Pump \\
\cline { 2 - 5 } & Number & $\%$ & Number & $\%$ \\
\hline Recent MI & 6 & $\mathbf{2 0}$ & 0 & $\mathbf{0}$ \\
\hline Old MI & 10 & $\mathbf{3 3 . 3 3}$ & 12 & $\mathbf{4 0 . 0 0}$ \\
\hline Previous MI (Total) & 16 & $\mathbf{5 3 . 3 3}$ & 12 & $\mathbf{4 0 . 0 0}$ \\
\hline
\end{tabular}

Table 5:- showing Comparison between preoperative laboratory data of the studied groups.

\begin{tabular}{|c|c|c|c|c|c|}
\hline \multirow[t]{2}{*}{ Preoperative } & \multicolumn{2}{|c|}{ On-pump } & \multicolumn{2}{|c|}{ Off-pump } & \multirow[t]{2}{*}{$\mathrm{P}$ value } \\
\hline & Mean & SD & Mean & SD & \\
\hline $\mathrm{Hb}_{\mathrm{g} / \mathrm{dl} \mathrm{l}}$ & 12.97 & 1.43 & 13.54 & 1.21 & 0.101 \\
\hline Ck-MB U/i & 22.20 & 7.27 & 22.27 & 6.95 & 0.973 \\
\hline Troponin $_{\mathrm{U} / \mathrm{i}}$ & 0.76 & 1.64 & 0.07 & 0.08 & $0.028 *$ \\
\hline $\mathrm{RBS}_{\mathrm{mg} / \mathrm{dl}}$ & 118.40 & 32.02 & 114.87 & 64.87 & 0.790 \\
\hline $\mathrm{ALT}_{\mathrm{U} / \mathrm{i}}$ & 28.03 & 10.90 & 31.70 & 10.77 & 0.195 \\
\hline $\mathrm{AST}_{\mathrm{U} / \mathrm{i}}$ & 26.20 & 9.43 & 27.90 & 7.47 & 0.442 \\
\hline Bil.(Total) $)_{\mathrm{m} / \mathrm{dl}}$ & 0.73 & 0.34 & 0.64 & 0.26 & 0.293 \\
\hline $\mathrm{K}_{\mathrm{mmol} / 1}$ & 4.47 & 0.40 & 4.79 & 0.51 & $0.009 *$ \\
\hline $\mathrm{Na}_{\text {mmol/1 }}$ & 140.53 & 2.96 & 141.27 & 3.86 & 0.412 \\
\hline $\mathrm{Cl}_{\mathrm{mmol} / 1}$ & 103.40 & 4.08 & 101.80 & 4.80 & 0.170 \\
\hline $\mathrm{Mg}_{\text {mmol/d1 }}$ & 0.98 & 0.17 & 1.01 & 0.29 & 0.561 \\
\hline $\mathrm{BUN}_{\mathrm{mg} / \mathrm{dl}}$ & 34.77 & 16.58 & 31.20 & 11.07 & 0.332 \\
\hline Creat $\mathrm{mg} / \mathrm{dl}$ & 1.87 & 0.24 & 1.87 & 0.22 & 0.933 \\
\hline Creat Cler $\mathrm{ml} / \mathrm{min}$ & 40.43 & 8.63 & 39.41 & 9.48 & 0.665 \\
\hline $\begin{array}{l}\text { Preop } \\
\text { Albuminuria } g / 24 \mathrm{~h}\end{array}$ & 1.367 & 1.180 & 1.33 & 1.20 & 0.902 \\
\hline
\end{tabular}


Table 6:- showing Comparison between preoperative echocardiographic data of the studied groups.

\begin{tabular}{|l|l|l|l|l|l|}
\hline Echocardiography & On-pump & Off-pump & P value \\
\cline { 2 - 6 } & Mean & SD & Mean & SD & \\
\hline Mean EF & 0.52 & 0.13 & 0.52 & 0.09 & 0.972 \\
\hline
\end{tabular}

Table 7:- showing preoperative vital signs among the studied groups

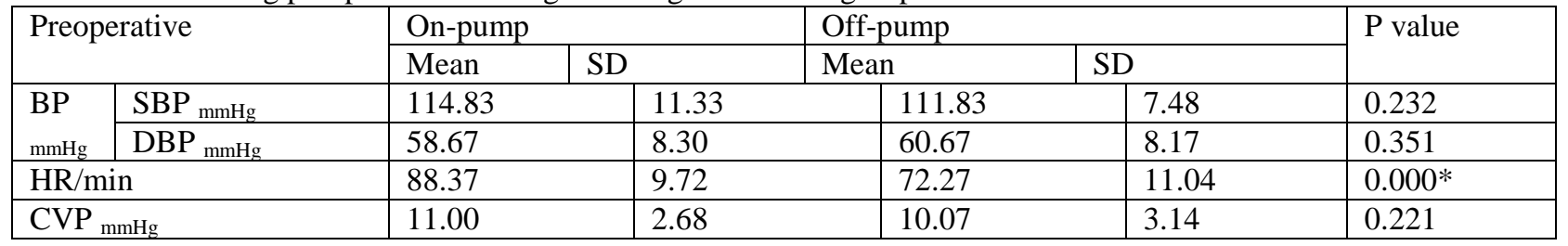

Table 8:- showing Abdominal and Pelvic U/S findings among studied groups.

\begin{tabular}{|l|l|l|l|l|}
\hline & On-pump & \multicolumn{2}{l|}{ Off-pump } \\
\hline Abdominal US & Number & $\%$ & Number & $\%$ \\
\hline Chronic renal insufficiency & 4 & 13.33 & 2 & 6.67 \\
\hline Chronic renal insufficiency grade I & 1 & 3.33 & - & 0.00 \\
\hline Chronic renal insufficiency grade II & 3 & 10.00 & 2 & 6.67 \\
\hline Chronic renal insufficiency grade III & 2 & 6.67 & - & 0.00 \\
\hline Chronic renal insufficiency, Rt renal cyst & 1 & 3.33 & - & 0.00 \\
\hline Compansated renal insufficiency & 1 & 3.33 & - & 0.00 \\
\hline Diabetic nephropathy & 2 & 6.67 & 8 & 26.67 \\
\hline $\begin{array}{l}\text { Diabetic nephropathy, } \\
\text { Chronic renal insufficiency grade II }\end{array}$ & 1 & 3.33 & - & 0.00 \\
\hline Non functioning shrunken Lt kidney & 1 & 3.33 & - & 0.00 \\
\hline Rt partial renal resection & 1 & 3.33 & - & 0.00 \\
\hline $\begin{array}{l}\text { Rt Renal artery stenosis, } \\
\text { Chronic renal insufficiency grade II }\end{array}$ & 1 & 3.33 & - & 0.00 \\
\hline Normal & 10 & 33.33 & 10 & 33.33 \\
\hline
\end{tabular}

Table 9:-showing operative data

\begin{tabular}{|l|l|l|l|l|l|}
\hline Preoperative & On-pump & Off-pump & P value \\
\cline { 2 - 5 } & Mean & SD & Mean & SD & \\
\hline Total pump Time min & 111.30 & 36.64 & & & \\
\hline Total ischemic Time min & 67.50 & 24.18 & & & \\
\hline Total OP Time min & 234.60 & 57.54 & 273.77 & 43.07 & $0.004 *$ \\
\hline No of Distal anasomoses. & 3.50 & 0.94 & 3.63 & 0.81 & 0.558 \\
\hline
\end{tabular}

Table 10:- showing need for inotropes and IAB

\begin{tabular}{|l|l|l|l|l|}
\hline \multirow{2}{*}{ IABP } & On - Pump & \multicolumn{2}{l|}{ Of - Pump } \\
\cline { 2 - 5 } & Number & $\%$ & Number & $\%$ \\
\hline \multirow{2}{*}{ Inotropes } & $3 / 30$ & $\mathbf{1 0 . 0 0}$ & $2 / 30$ & $\mathbf{6 . 6 7}$ \\
\hline & On - Pump & Of - Pump & $\%$ \\
\cline { 2 - 5 } & Number & $\%$ & Number & $\mathbf{9 0 . 0 0}$ \\
\hline
\end{tabular}


Table 11:- showing post operative laboratory data

\begin{tabular}{|c|c|c|c|c|c|}
\hline \multirow[t]{2}{*}{ Postoperative } & \multicolumn{2}{|c|}{ On-pump } & \multicolumn{2}{|c|}{ Off-pump } & \multirow[t]{2}{*}{$\mathrm{P}$ value } \\
\hline & Mean & SD & Mean & SD & \\
\hline $\mathrm{Hg} \mathrm{g/dl}$ & 9.62 & 1.10 & 10.35 & 0.89 & $0.006 *$ \\
\hline $\mathrm{Ck}-\mathrm{MB}_{\mathrm{U} / \mathrm{i}}$ & 45.61 & 24.07 & 45.27 & 27.78 & 0.960 \\
\hline Troponin $_{\mathrm{U} / \mathrm{i}}$ & 4.26 & 4.57 & 1.77 & 1.48 & $0.007 *$ \\
\hline $\mathrm{RBS}_{\mathrm{mg} / \mathrm{dl}}$ & 155.43 & 57.27 & 133.73 & 39.91 & 0.095 \\
\hline $\mathrm{ALT}_{\mathrm{U} / \mathrm{i}}$ & 26.63 & 16.74 & 30.73 & 12.09 & 0.282 \\
\hline $\mathrm{AST}_{\mathrm{U} / \mathrm{i}}$ & 65.40 & 35.19 & 59.83 & 32.98 & 0.530 \\
\hline Bil.(Total) $\mathrm{mg} / \mathrm{dl}$ & 0.79 & 0.46 & 0.90 & 0.29 & 0.289 \\
\hline $\mathrm{K}_{\mathrm{mmol} / 1}$ & 5.22 & 0.62 & 5.06 & 0.38 & 0.236 \\
\hline $\mathrm{Na}_{\mathrm{mmol} / 1}$ & 146.40 & 4.61 & 144.80 & 5.67 & 0.235 \\
\hline $\mathrm{Cl}_{\mathrm{mmol} / 1}$ & 109.93 & 4.73 & 110.67 & 5.36 & 0.577 \\
\hline $\mathrm{Mg}_{\mathrm{mmol} / \mathrm{dl}}$ & 1.32 & 0.30 & 1.22 & 0.25 & 0.170 \\
\hline $\mathrm{BUN}_{\mathrm{mg} / \mathrm{dl}}$ & 48.07 & 15.36 & 32.40 & 15.33 & $0.000^{*}$ \\
\hline Creat $\mathrm{mg} / \mathrm{dl}$ & 2.60 & 0.87 & 1.96 & 0.65 & $0.002 *$ \\
\hline Creat Cler $\mathrm{ml} / \mathrm{min}$ & 31.57 & 11.04 & 39.94 & 13.68 & $0.012 *$ \\
\hline $\begin{array}{l}\text { Post op } \\
\text { Albuminuria } g / 24 \mathrm{~h}\end{array}$ & 1.079 & 0.828 & 1.32 & 1.23 & 0.370 \\
\hline
\end{tabular}

Table 12:- showing post operative vital signs

\begin{tabular}{|c|c|c|c|c|c|c|}
\hline \multirow{2}{*}{\multicolumn{2}{|c|}{ postoperative }} & \multicolumn{2}{|c|}{ On-pump } & \multicolumn{2}{|c|}{ Off-pump } & \multirow[t]{2}{*}{$\mathrm{P}$ value } \\
\hline & & Mean & SD & Mean & SD & \\
\hline \multirow{2}{*}{$\begin{array}{l}\mathrm{BP} \\
\mathrm{mmHg}\end{array}$} & $\mathrm{SBP}_{\mathrm{mmHg}}$ & 123.00 & 16.64 & 125.00 & 11.96 & 0.595 \\
\hline & $\mathrm{DBP}_{\mathrm{mmHg}}$ & 57.67 & 8.17 & 63.67 & 8.90 & $0.009 *$ \\
\hline \multicolumn{2}{|c|}{$\mathrm{HR} / \mathrm{min}$} & 90.87 & 12.21 & 84.23 & 7.90 & $0.016^{*}$ \\
\hline \multicolumn{2}{|c|}{$\mathrm{CVP}_{\mathrm{mmHg}}$} & 10.17 & 4.44 & 9.33 & 3.20 & 0.408 \\
\hline
\end{tabular}

Table 13:- showing comparion between need for drug support

\begin{tabular}{|l|l|l|l|l|l|}
\hline \multirow{2}{*}{ postoperative } & On-pump & Off-pump & \multirow{2}{*}{ P value } \\
\cline { 2 - 5 } & Mean & SD & Mean & SD & \multirow{2}{*}{0.195} \\
\hline $\begin{array}{l}\text { Vasopressor } \\
\text { (Noradrenalin) } \mathrm{ug} / \mathrm{kg} / \mathrm{min}\end{array}$ & 0.08 & 0.09 & 0.05 & 0.08 & $0.012^{*}$ \\
\hline Diuretics (Frusimide) $\mathrm{mg}$ & 63.70 & 41.52 & 38.75 & 25.76 & 0.087 \\
\hline Bl. Trnsfusion ml & 735.33 & 495.71 & 480.00 & 238.90 & 0.953 \\
\hline Urine Output $\mathrm{ml} / 24$ & 3938.33 & 1056.07 & 3918.33 & 1512.11 & \\
\hline
\end{tabular}

Table 14:- showing ICU stay in days

\begin{tabular}{|l|l|l|l|l|l|}
\hline \multirow{2}{*}{} & \multicolumn{2}{|l|}{ On-pump } & Off-pump & P value \\
\cline { 2 - 6 } & Mean & SD & Mean & SD & \\
\hline ICU stay (days) & 4.667 & 3.367 & 3.70 & 2.15 & 0.191 \\
\hline
\end{tabular}

Table 15:- showing complications between groups

\begin{tabular}{|l|l|l|l|l|}
\hline \multirow{2}{*}{} & On-pump & Off-pump & $\%$ \\
\cline { 2 - 5 } & Number & $\%$ & Number & 10.00 \\
\hline Arrhythmias & 3 & 10.00 & 3 & 30.00 \\
\hline Re-opening & 7 & 23.33 & 9 & 16.67 \\
\hline Dialysis & 2 & 6.67 & 5 & 10.00 \\
\hline
\end{tabular}


Table 16:- showing Preoperative and postoperative kidney functions in On-pump group.

\begin{tabular}{|l|l|l|l|l|l|l|l|l|}
\hline $\begin{array}{l}\text { Patient } \\
\text { Number }\end{array}$ & $\begin{array}{l}\text { preOp. } \\
\text { Creat. } \\
\text { g//dl }\end{array}$ & $\begin{array}{l}\text { PostOp. } \\
\text { Creat. } \\
\text { M/d/ll }\end{array}$ & $\begin{array}{l}\text { PreOp. } \\
\text { Creat.Clear. } \\
\text { m/min }\end{array}$ & $\begin{array}{l}\text { PostOp. } \\
\text { Creat.Clear. } \\
\text { m/min }\end{array}$ & $\begin{array}{l}\text { PreOp. } \\
\text { BUN } \\
\text { mg/dl }\end{array}$ & $\begin{array}{l}\text { PostOp. } \\
\text { BUN } \\
\text { mg/dl }\end{array}$ & $\begin{array}{l}\text { PreOp. } \\
\text { Alb. } \\
\text { g/24h }\end{array}$ & $\begin{array}{l}\text { PostOp. } \\
\text { Alb. } \\
\text { g/24h }\end{array}$ \\
\hline 1 & 1.66 & 1.88 & 48 & 42.4 & 23 & 38 & 0.56 & 0.54 \\
\hline 2 & 1.79 & 1.28 & 40 & 56 & 27 & 22 & 0.39 & 0.53 \\
\hline 3 & 1.7 & 3.13 & 30.6 & 16.6 & 26 & 52 & 2.01 & 1.12 \\
\hline 4 & 1.99 & 1.98 & 29.7 & 29.8 & 38 & 41 & 1.21 & 1.17 \\
\hline 5 & 2.14 & 2.8 & 40 & 30.5 & 32 & 61 & 2.41 & 2.03 \\
\hline 6 & 2.06 & 3.34 & 42 & 25.9 & 31 & 65 & 2.82 & 1.94 \\
\hline 7 & 1.61 & 3.4 & 50.7 & 24 & 23 & 66 & 3.31 & 1.76 \\
\hline 8 & 1.75 & 2.76 & 51.5 & 32.7 & 24 & 52 & 3.42 & 1.81 \\
\hline 9 & 2.07 & 1.79 & 25.6 & 29.6 & 46 & 42 & 1.26 & 1.34 \\
\hline 10 & 1.91 & 2.56 & 30.23 & 22.5 & 39 & 46 & 0.3 & 0.41 \\
\hline 11 & 2.3 & 2.45 & 41.2 & 38.7 & 99 & 57 & 2.3 & 2.18 \\
\hline 12 & 1.6 & 1.61 & 32.9 & 32.8 & 29 & 35 & 1.7 & 1.89 \\
\hline 13 & 1.7 & 1.39 & 51.3 & 62.7 & 27 & 26 & 2.7 & 2.96 \\
\hline 14 & 1.64 & 1.68 & 45.8 & 44.7 & 21 & 34 & 0.9 & 0.98 \\
\hline 15 & 1.61 & 1.67 & 46.5 & 44.8 & 27 & 23 & 0.22 & 0.44 \\
\hline 16 & 1.93 & 2.1 & 36.1 & 33.4 & 29 & 31 & 0.017 & 0.02 \\
\hline 17 & 2.28 & 3.14 & 31.9 & 23.22 & 62 & 80 & 0.41 & 0.47 \\
\hline 18 & 1.78 & 2.23 & 42.4 & 33.8 & 37 & 42 & 0.11 & 0.19 \\
\hline 19 & 1.64 & 2.87 & 33.1 & 18.9 & 27 & 50 & 1.62 & 1.09 \\
\hline 20 & 1.94 & 2.53 & 34.8 & 26.7 & 39 & 46 & 0.46 & 0.4 \\
\hline 21 & 1.85 & 3.1 & 41.2 & 24.6 & 26 & 51 & 2.6 & 1.57 \\
\hline 22 & 1.87 & 2.32 & 36.3 & 29.3 & 35 & 42 & 1.96 & 1.59 \\
\hline 23 & 1.64 & 2.95 & 45.27 & 25.2 & 19 & 49 & 0.03 & 0.1 \\
\hline 24 & 1.64 & 1.69 & 45.73 & 44.37 & 13 & 26 & 0.05 & 0.04 \\
\hline 25 & 1.7 & 4.27 & 36.1 & 14.38 & 23 & 63 & 0.019 & 0.014 \\
\hline 26 & 2.02 & 4.44 & 67.8 & 30.8 & 42 & 70 & 3.7 & 1.99 \\
\hline 27 & 2.1 & 4.16 & 39.3 & 19.8 & 58 & 57 & 0.39 & 0.3 \\
\hline 28 & 2.5 & 3.99 & 36 & 22.55 & 49 & 63 & 1.82 & 1.29 \\
\hline 29 & 2.01 & 2.03 & 35.2 & 34.9 & 46 & 39 & 2.29 & 2.2 \\
\hline 30 & 1.77 & 2.56 & 45.7 & 31.6 & 26 & 73 & 0.02 & 0.018 \\
\hline
\end{tabular}

Table 17:- showing Preoperative and postoperative kidney functions in Off-pump group.

\begin{tabular}{|l|l|l|l|l|l|l|l|l|}
\hline $\begin{array}{l}\text { Patient } \\
\text { Number }\end{array}$ & $\begin{array}{l}\text { preOp. } \\
\text { Creat. } \\
\text { Mg/dl }\end{array}$ & $\begin{array}{l}\text { PostOp. } \\
\text { Creat. } \\
\text { Mg/dl }\end{array}$ & $\begin{array}{l}\text { PreOp. } \\
\text { Creat.Clear. } \\
\text { m/min }\end{array}$ & $\begin{array}{l}\text { PostOp. } \\
\text { Creat.Clear. } \\
\text { m//min }\end{array}$ & $\begin{array}{l}\text { PreOp. } \\
\text { BUN } \\
\text { m/dld }\end{array}$ & $\begin{array}{l}\text { PostOp. } \\
\text { BUN } \\
\text { mg/dl }\end{array}$ & $\begin{array}{l}\text { PreOp. } \\
\text { Alb. } \\
\mathrm{g} / 24 \mathrm{~h}\end{array}$ & $\begin{array}{l}\text { PostOp. } \\
\text { Alb. } \\
\mathrm{g} / 24 \mathrm{~h}\end{array}$ \\
\hline 1 & 2.4 & 4.85 & 31.25 & 15.46 & 71 & 93 & 0.96 \\
\hline 2 & 1.84 & 1.95 & 39.9 & 37.7 & 22 & 25 & 0.11 & 0.1 \\
\hline 3 & 1.61 & 1.35 & 51.5 & 61.48 & 18 & 18 & 0.02 & 0.015 \\
\hline 4 & 1.78 & 1.57 & 36.86 & 41.79 & 26 & 22 & 0.31 & 0.51 \\
\hline 5 & 2.18 & 2.37 & 38.7 & 35.63 & 35 & 43 & 2.01 & 1.82 \\
\hline 6 & 1.63 & 1.7 & 74.13 & 71 & 30 & 27 & 3.9 & 3.86 \\
\hline 7 & 1.69 & 1.58 & 33.13 & 35.4 & 36 & 29 & 0.46 & 0.42 \\
\hline 8 & 1.97 & 2.21 & 32 & 28.5 & 28 & 33 & 2.32 & 2.13 \\
\hline 9 & 1.76 & 1.38 & 48.96 & 62.44 & 23 & 19 & 3.5 & 3.86 \\
\hline 10 & 1.85 & 2.36 & 31.71 & 24.8 & 29 & 41 & 2.54 & 2.15 \\
\hline 11 & 2.05 & 1.85 & 36.5 & 40.5 & 31 & 29 & 1.6 & 1.94 \\
\hline 12 & 1.86 & 1.92 & 34 & 32.98 & 27 & 28 & 0.1 & 0.13 \\
\hline 13 & 2.16 & 2.81 & 27 & 20.8 & 39 & 49 & 1.54 & 1.19 \\
\hline 14 & 1.77 & 1.72 & 36 & 37.13 & 29 & 28 & 0.02 & 0.017 \\
\hline 15 & 1.66 & 1.82 & 43.87 & 40 & 26 & 29 & 2.66 & 2.45 \\
\hline
\end{tabular}




\begin{tabular}{|l|l|l|l|l|l|l|l|l|}
\hline 16 & 1.72 & 1.67 & 35 & 36.13 & 22 & 20 & 0.2 & 0.25 \\
\hline 17 & 1.68 & 1.74 & 41.5 & 40.08 & 19 & 19 & 0.1 & 0.14 \\
\hline 18 & 1.65 & 2 & 19.16 & 15.8 & 36 & 40 & 0.015 & 0.01 \\
\hline 19 & 1.78 & 1.6 & 42.6 & 46.86 & 28 & 24 & 0.25 & 0.3 \\
\hline 20 & 2.23 & 2.04 & 41.77 & 45.66 & 43 & 35 & 2.52 & 2.84 \\
\hline 21 & 1.98 & 2.12 & 35.9 & 33.54 & 25 & 32 & 1.86 & 1.79 \\
\hline 22 & 2.35 & 2.69 & 45.47 & 39.7 & 51 & 65 & 2.7 & 2.43 \\
\hline 23 & 1.76 & 1.82 & 34.6 & 33.4 & 34 & 36 & 1.7 & 1.58 \\
\hline 24 & 1.62 & 1.42 & 40.46 & 46.16 & 23 & 15 & 0.3 & 0.38 \\
\hline 25 & 1.82 & 2.07 & 41.1 & 35.6 & 29 & 36 & 2.3 & 2.17 \\
\hline 26 & 2.1 & 1.85 & 35 & 39.8 & 34 & 31 & 0.4 & 0.48 \\
\hline 27 & 1.7 & 1.92 & 40.27 & 35.66 & 18 & 28 & 0.61 & 0.52 \\
\hline 28 & 1.82 & 1.71 & 42.43 & 45.16 & 25 & 22 & 0.2 & 0.24 \\
\hline 29 & 1.65 & 1.46 & 39.56 & 44.7 & 30 & 29 & 1 & 1.15 \\
\hline 30 & 1.98 & 1.39 & 52 & 74.2 & 49 & 27 & 3.2 & 3.91 \\
\hline
\end{tabular}

Table 18:-showing Cleveland foundation score for postoperative renal dysfunction patients in on-pump and offpump groups applied to our study.

\begin{tabular}{|c|c|c|c|c|c|c|c|}
\hline \multirow{2}{*}{$\begin{array}{l}\text { Patient } \\
\text { Number }\end{array}$} & \multicolumn{7}{|c|}{ Parameters } \\
\hline & $\begin{array}{l}\text { Female } \\
\text { gender }\end{array}$ & $\begin{array}{l}\text { EF } \\
<35 \%\end{array}$ & IABP & DM & $\begin{array}{l}\text { Creat } 1.2 \text { to } \\
<2.1\end{array}$ & $\begin{array}{l}\text { Creat } \geq 2.1 \\
\mathrm{Mg} / \mathrm{dl}\end{array}$ & Total \\
\hline \multicolumn{8}{|c|}{ On-pump } \\
\hline 3 & 1 & $\mathbf{0}$ & $\mathbf{0}$ & 1 & 2 & $\mathbf{0}$ & 4 \\
\hline 6 & $\mathbf{0}$ & $\mathbf{0}$ & $\mathbf{0}$ & 1 & 2 & $\mathbf{0}$ & 3 \\
\hline 7 & $\mathbf{0}$ & 1 & 2 & 1 & 2 & (0) & 6 \\
\hline 8 & $\mathbf{0}$ & 0 & $\mathbf{0}$ & 1 & 2 & $\mathbf{0}$ & 3 \\
\hline 17 & $\mathbf{0}$ & 1 & 2 & $\mathbf{0}$ & $\mathbf{0}$ & 5 & 8 \\
\hline 19 & 1 & 0 & $\mathbf{0}$ & 1 & 2 & $\mathbf{0}$ & 2 \\
\hline 21 & $\mathbf{0}$ & 0 & $\mathbf{0}$ & 1 & 2 & $\mathbf{0}$ & 2 \\
\hline 23 & $\mathbf{0}$ & $\mathbf{0}$ & $\mathbf{0}$ & $\mathbf{0}$ & 2 & $\mathbf{0}$ & 2 \\
\hline 25 & $\mathbf{0}$ & $\mathbf{0}$ & $\mathbf{0}$ & $\mathbf{0}$ & 2 & 0 & 2 \\
\hline 26 & $\mathbf{0}$ & 0 & $\mathbf{0}$ & 1 & 2 & $\mathbf{0}$ & 3 \\
\hline 27 & $\mathbf{0}$ & $\mathbf{0}$ & $\mathbf{0}$ & $\mathbf{0}$ & 2 & $\mathbf{0}$ & 2 \\
\hline 28 & $\mathbf{0}$ & $\mathbf{0}$ & $\mathbf{0}$ & 1 & $\mathbf{0}$ & 5 & 6 \\
\hline 30 & 1 & $\mathbf{0}$ & $\mathbf{0}$ & $\mathbf{0}$ & 2 & $\mathbf{0}$ & 3 \\
\hline \multicolumn{8}{|c|}{ Off -pump } \\
\hline 1 & $\mathbf{0}$ & 0 & $\mathbf{0}$ & 1 & $\mathbf{0}$ & 5 & 6 \\
\hline 13 & $\mathbf{0}$ & 1 & $\mathbf{0}$ & 1 & $\mathbf{0}$ & 5 & 7 \\
\hline 15 & 1 & $\mathbf{0}$ & 0 & 1 & 0 & 2 & 7 \\
\hline
\end{tabular}


Table 19:- showing Variable risk factors in patients who required dialysis in the on-pump group.

\begin{tabular}{|c|c|c|c|c|c|c|c|c|c|c|c|c|}
\hline $\begin{array}{l}\text { Patient } \\
\text { numbe } \\
\mathrm{r}\end{array}$ & $\begin{array}{l}\text { Gende } \\
\mathbf{r}\end{array}$ & $\begin{array}{l}\text { Ag } \\
\text { e }\end{array}$ & $\begin{array}{l}\text { HT } \\
\mathbf{N}\end{array}$ & $\begin{array}{l}\text { D.M } \\
\text { - }\end{array}$ & $\begin{array}{l}\text { M. } \\
\text { I }\end{array}$ & $\mathbf{E F}$ & $\mathbf{U} / \mathbf{S}$ & $\begin{array}{l}\text { Crea } \\
t\end{array}$ & $\begin{array}{l}\text { Pum } \\
\text { p } \\
\text { time }\end{array}$ & $\begin{array}{l}\text { Vasopressor } \\
\text { s }\end{array}$ & $\begin{array}{l}\text { M. } \\
\text { I }\end{array}$ & $\begin{array}{l}\text { REope } \\
\text { n }\end{array}$ \\
\hline 3 & $\mathrm{~F}$ & 72 & $\mathrm{Y}$ & $\mathrm{Y}$ & $\mathrm{Y}$ & $\begin{array}{l}63 \\
\%\end{array}$ & $\begin{array}{l}\text { RAS } \\
\text { NEP } \\
\text { H } \\
\text { II }\end{array}$ & 1.7 & 155 & 0.04 & $\mathrm{~N}$ & $\mathrm{~N}$ \\
\hline 7 & $\mathrm{M}$ & 70 & $\mathrm{Y}$ & $\mathrm{Y}$ & $\mathrm{Y}$ & $\begin{array}{l}30 \\
\%\end{array}$ & NAD & 1.61 & 210 & 0.12 & $\mathrm{~N}$ & $\mathrm{~N}$ \\
\hline 21 & $\mathrm{M}$ & 73 & $\mathrm{Y}$ & $\mathrm{Y}$ & $\mathrm{N}$ & $\begin{array}{l}60 \\
\%\end{array}$ & $\begin{array}{l}\text { NEP } \\
\mathrm{H} \\
\mathrm{II}\end{array}$ & 1.85 & 70 & 0.24 & $\mathrm{~N}$ & $\mathrm{Y}$ \\
\hline 23 & $\mathrm{M}$ & 74 & $\mathrm{Y}$ & $\mathrm{N}$ & $\mathrm{N}$ & $\begin{array}{l}65 \\
\%\end{array}$ & NAD & 1.64 & 155 & 0.04 & $\mathrm{Y}$ & $\mathrm{Y}$ \\
\hline 26 & $\mathrm{M}$ & 55 & $\mathrm{Y}$ & $\mathrm{N}$ & $\mathrm{Y}$ & $\begin{array}{l}45 \\
\%\end{array}$ & $\begin{array}{l}\text { NEP } \\
\mathrm{H} \\
\mathrm{II}\end{array}$ & 2.02 & 90 & 0.04 & $\mathrm{Y}$ & $\mathrm{N}$ \\
\hline 27 & $\mathrm{M}$ & 80 & $\mathrm{Y}$ & $\mathrm{N}$ & $\mathrm{N}$ & $\begin{array}{l}70 \\
\%\end{array}$ & NAD & 2.1 & 120 & 0.08 & $\mathrm{Y}$ & $\mathrm{N}$ \\
\hline
\end{tabular}

Table 20:- showing Variable risk factors in patients who required dialysis in the off-pump group.

\begin{tabular}{|l|l|l|l|l|l|l|l|l|l|l|l|}
\hline $\begin{array}{l}\text { Patient } \\
\text { number }\end{array}$ & $\begin{array}{l}\text { Gende } \\
\text { r }\end{array}$ & Age & HTN & D.M. & M.I & EF & U/S & Creat & Vasopressors & M.I & Reopen \\
\hline 1 & $\mathrm{M}$ & 80 & $\mathrm{Y}$ & $\mathrm{Y}$ & $\mathrm{Y}$ & $45 \%$ & $\begin{array}{l}\text { NEPH } \\
\text { II }\end{array}$ & 2.4 & 0.04 & $\mathrm{~N}$ & $\mathrm{Y}$ \\
\hline 13 & $\mathrm{M}$ & 86 & $\mathrm{Y}$ & $\mathrm{Y}$ & $\mathrm{Y}$ & $35 \%$ & $\mathrm{NEPH}$ & 2.16 & 0.04 & $\mathrm{~N}$ & $\mathrm{~N}$ \\
\hline 15 & $\mathrm{~F}$ & 71 & $\mathrm{Y}$ & $\mathrm{Y}$ & $\mathrm{Y}$ & $40 \%$ & $\mathrm{NAD}$ & 1.66 & 0.02 & $\mathrm{~N}$ & $\mathrm{Y}$ \\
\hline
\end{tabular}

\section{Discussion:-}

\section{Risk Factors other than renal Insufficiency and preoprative data:-}

Analysis of the tables shows no significant difference in preoperative data and in risk factors percentages( smoking, hypertension,DM, Dyslipidemia, and previous MI) in both groups which indicates that no preference was made for any risk factor on aiming at off-pump as long as the patient was fulfilling the inclusion criteria for our study. In our study the percentage of hypertensive patients in both groups are higher by $10 \%$ up to $40 \%$ compared to some papers which as discussed below raised our attention towards the quality of our screening programs for hypertensive patients and recommending more follow up of such patients and these recommendations were raised to mortality and morbidity committee in Measurement of glycosylated haemoglobin [1] (HbA1c) and admission of patients with levels $<6 \%$ for the operation, and delaying the operation if $\mathrm{HbA} 1 \mathrm{c} \geq 6 \%$ is recommended. As $\mathrm{HbA} 1 \mathrm{c} \geq 6 \%$ was associated with an increased risk of postoperative superficial sternal wound infections and a trend for higher mediastinitis rate and significantly higher mortality three years after CABG[12]

\section{Preoperative abdominal and pelvic U/S:-}

Analysis of the preoperative abdominal and pelvic U/S shows there was no statistically significant difference between the two groups as 10 patients had normal U/S examination in both groups. As for the other findings, these are not considered to be risk factors for renal impairment postoperatively as Ultrasound of the kidneys is a useful screening tool for kidney stones, cysts and masses. It can assess complications of obstructive kidneys stones. It provides useful information when a patient is in renal failure or if there is blood found in the urine. It can also provide information for those suffering from repeated urinary tract infections. But it's not considered a renal function test.[13] Analysis of post operative results (as will be discussed later) showed no correlation between preoperative finding of abdomio-pelvic U/S and postoperative renal impairment.

\section{Operative Data:-}

Pump time: Comparing our pump time to different studies showed that our patients stayed for a longer time on the $\mathrm{CPB}$, however, when this is plotted against number of distal anastomoses we have mean of 3.5 distal anastomoses / 
patient compared to Loef,et al[14]., Pramodh, et al.[15], Vedin.[16], and Ascione, et al [17]. that were less than 3. The more prolonged total time of surgery in the OPCAB group can be attributed to the relatively more difficult technical demands of the technique and the fact that before every anastomosis there is some time needed to stabilize the heart without compromising the heamodynamics. It also worth mentioning here that there was no incident of hemodynamic instability at any of our patient of the OPCAB group necessitating conversion to on-pump technique.

\section{Number of distal Anastomoses:-}

Analysis of the table shows no significant difference in number of distalan astomoses in both groups which indicates that no preference was made on aiming at off-pump as long as the patient was fulfilling the inclusion criteria forour study.

\section{Need for IABP:-}

Although slightly more patients in our study needed IABP support but the results are just slightly higher. Noted also that in the mentioned studies, including ours, more patients in the on-pump group needed IABP. In the On-pump group, the 3 patients who needed IABP were: Patient number 7: EF was 30\%, Patient number 14: EF was 30\%, Patient number 17: EF was $28 \%$ In the Off-pump group, the 2 patients who needed IABP were: Patient number 21: EF was $40 \%$, Patient number 24: EF was $45 \%$

So although more patients in the on-pump group needed IABP but these patients were more critical than the patients in the off-pump group with respect to EF criteria.

\section{Need for Inotropes:-}

In our study, few more patients in the on-pump group were put on inotropic support than in the off-pump group, intraoperatively. In other studies much more patients in the on-pump group were put on inotropic support, that may be to overcome periods of stunning after CPB. But there is a huge difference between the percentages of our total number of patients who were put on inotropic support in both groups when compared to other studies; this is due to the anesthesia protocol utilized in our center to transfer the patients on low dose inotropic support to the ICU.

\section{Post Operative Data:-}

Analysis of the postoperative laboratory results among the two groups shows there was no statistically significant difference between the two groups except for: - The Hemoglobin, which is lower in on-pump group due to hemodilution effect of CPB - The troponin, as we discussed before, the 6 patients who had recent infarction we preferred to do them on-pump. - Kidney function tests, this will be discussed separately in details later.

\section{Postoperative Vital Signs:}

Analysis of the postoperative vital signs among the two groups shows there was no statistically significant difference between the two groups except for: - The Heart Rate: lower in the off-pump group, this can be attributed to the usage of higher doses of inotropic support in the on-pump goup. - The Diastolic Blood pressure: lower in the on-pump group, this is due to post $\mathrm{CPB}$ vasodilatation as the vasoconstriction present during the CPB gives way to vasodilation after CPB has been discontinued, and particularly after the administration of protamine.[18]

\section{Postoperative need for blood transfusion:-}

Although there is no statistical significant difference between the two groups, on-pump group patients needed higher amounts of blood transfusion than off-pump patients in our study and in other studies, this is could be attributed to coagulopathic effect of CPB.

\section{Complications other than Dialysis:- Re-opening for bleeding:-}

Our results are different from other studies regarding the rate of reopening for bleeding in the off-pump group which is even much higher than in our on-pump group. Moreover, both of our groups had a higher rate of reopening than other studies. We failed to explain this phenomenon. However, and due to these results, more attention were paid to our haemostasis and wound closure techniques.

IABP post operative: Apart from patients who needed IABP support intraoperatively: 
- In the on-pump group, patient number 23 was complicated with AF and MI resulting in hemodynamic instability necessitating IABP support for 96 hours. - In the off-pump group patient number 10 was complicated with AF and MI resulting in hemodynamic instability necessitating IABP support for 48 hours.

Myocardial Infarction: Despite the technical precautions, newly developed postoperative ECG ischemic changes and elevated cardiac enzymes complicated the outcome of 3 patients in the on-pump group and 3 in the off-pump group. The incidence of postoperative MI in our study is comparable to other studies and it is also noted that there was no difference between both on and off-pump groups.

Arrhythmias:-In the on-pump group 7 patients were complicated with Arrhythmias asfollows:o Patient number 2 with 1st degree AV block. o Patients number 5, 7, 15, 19,23,27 with Atrial Fibrillation. In the off-pump groups 9 patients were complicated with Arrhythmias as follows:o Patients number 1, 7, 10, 14, 18, 23, 28 with Atrial Fibrillation. o Patient numbers 12, 25 with Ventricular Extra systoles. The incidence of postoperative AF in our study is comparable to other studies and it is also noted that there was no difference between both on and offpump groups.

ICU stay:-There is no statistical significant difference in ICU stay between the on-pump group and off-pump group in our study. Also noted that our ICU stay is much longer than other studies, this is due to our ICU protocol of Patient discharge. Comparison between pre and postoperative vital signs in on-pump group.

\section{Renal Dysfunction:-}

According to ACC/AHA 2004 Guideline Update for Coronary Artery Bypass Graft Surgery, the first major multicenter study of renal dysfunction after CABG surgery was published in 1998. This study of 2222 patients who underwent myocardial revascularization with CPB defined postoperative renal dysfunction (PRD) as a postoperative serum creatinine level of greater than or equal to $2.0 \mathrm{mg} / \mathrm{dL}$ or an increase in the serum creatinine level of greater than or equal to $0.7 \mathrm{mg} / \mathrm{dL}$ from preoperative to maximum postoperative values.[19] In on-pump group According to the criteria mentioned in the guidelines we have 13 patients in the on-pump group Vs only one patient in the offpump group diagnosed as (PRD) Postoperative Renal Dysfunction. These previous tables and graphs showed that in the 13 patients in the on-pump group and the one patient in the off-pump group who were diagnosed as having PRD, according to the increase between pre and postoperative serum creatinine levels, showed incremental decline in other kidney function tests as noted with levels of BUN and Creatinine clearance but no notable change in albuminurea levels. PRD Patients who required dialysis: In the on-pump group, there were 6 patients who required dialysis. These patients were number: $3,7,21,23,26,27$ and they were among the group of 13 patients whom were diagnosed as PRD. So the questions is why these 6 patients especially? Another question, can we predict patients who will require dialysis? The same applies for the off-pump group, where only 3 patients required dialysis (patients number $1,13,15)$ but only 1 was diagnosed as suffering PRD so again stay the same questions. To answer these questions we will compare the 6 patients in the on-pump group to the rest of the 13 patients, and we will examine the 3 patients in the off-pump group this will be done via plotting these patients in the Cleveland Clinic Foundation Acute Renal Failure Scoring System[20] Cleveland Clinic Foundation Acute Renal Failure Scoring System: • Female gender--1 1 Congestive heart failure-- $2 \cdot$ LV ejection fraction $<35 \%$-- 1 - Preoperative use of IABP-- 2 • COPD-- $1 \cdot$ Insulin-requiring diabetes-- $1 \cdot$ Previous cardiac surgery-- $1 \cdot$ Emergency surgery-- $2 \cdot$ Valve surgery only (reference to CABG)-- $1 \cdot \mathrm{CABG}+$ valve (reference to $\mathrm{CABG}$ )-- $2 \cdot$ Other cardiac surgeries-- 2 - Preoperative creatinine 1.2 to $<2.1 \mathrm{mg} / \mathrm{dl}--2 \cdot$ Preoperative creatinine $\geq 2.1-5$ For the 3 off-pump patients the results came as they were supposed to be and this scoring system was valid to predict postoperative renal dysfunction in this group. BUT, in the on-pump group the results were not accurate in predicting PRD, which could be attributed to the following:[21] 1. There is no parameter in this scoring system to consider the various pathophysiological parameters of the CPB. 2. Throughout the literature there is a strong agreement that multiple risk factors and multiple pathological and physiological factors interact together to affect the kidney functions such as: a. Preoperative factors: i. Lack of renal reserve. ii. Renovascular disease. iii. Prerenal azotemia. iv. Recent diuresis. v. NPO status. vi. Impaired LV function. vii. ACEI / ARB. viii. Nephrotoxins. ix. Intravenous contrast. x. Other medications. xi. Endotoxemia. xii. Inflammation.b. Intraoperative: i. Decreased renal perfusion. ii. Hypotension. iii. Lack of pulsatile flow. iv. Vasoactive agents. v. Anesthetic effects. vi. Embolic events. vii. CPB-induced Inflammation. viii. Nephrotoxins. ix. Hemolysis. c. Post Operative: x. Systemic inflammation. xi. Reduced LV function. xii. Vasoactive agents. xiii. Hemodynamic instability. xiv. Nephrotoxins. xv. Volume depletion. xvi. Sepsis. 
Another study suggested also that the following are risk factors: $\bullet$ Age $(\mathrm{yr}) . \cdot$ Weight $(\mathrm{kg})$. $\bullet$ Preoperative creatinine $(\mathrm{mg} / \mathrm{dl})$. C Cardiopulmonary bypass time (minutes).[20]

From the above there is no single accurate investigation that can predict renal function outcome after cardiac surgery in general, and this notion gained acceptance in the literature as we discussed before in the previous chapters of this current study, but still to validate this hypothesis we have to discuss each patient whom required dialysis separately.

The on-pump group patients. Analysis of the previous two tables strongly confirms the interaction of multiple risk factors on the outcome of renal affection. In most of the reviewed studies including ours, renal function is better preserved in patients undergoing off-pump CABG than those undergoing onpump CABG. Also, its notable that, in our study, there is higher percentage of patients who developed PRD requiring dialysis postoperatively than in the other different studies, especially in the on-pump group but this is expected as the criteria of inclusion for all our patients was "patients with preoperative renal impairment (serum creatinine 1.6-2.5 mg/dl)" but some of these studies included patients with normal serum creatinine preoperatively and measured the affection beyond normal renal function, and as we mentioned before the preoperative renal impairment is considered by itself a risk factor for postoperative renal dysfunction.

General Measures to prevent renal dysfunction after Cardiac Surgery:-

Identification of High-Risk Patients. In patients who undergo cardiac surgery, identifying patients who are at high risk for ARF is critically important. The important risk factors and scoring systems that can be used for this identification purpose have been discussed before.[21] Optimization of renal perfusion and avoidance of nephrotoxins.[21] Factors that alter renal blood flow and lead to prerenal azotemia should be identified and corrected. Treatment of volume depletion and congestive heart failure before cardiac surgery will increase cardiac output and renal perfusion. Perioperative hydration and the use of hemodynamic monitoring and inotropic agents to optimize cardiac output may be necessary. It is unknown whether intraoperative optimization of bypass flow, perfusion pressure, and oxygen delivery would affect the subsequent development of renal dysfunction, although conceptually this would seem to be a reasonable goal. Medications such as NSAID and other nephrotoxic agents should be discontinued. Whether ACEI and ARB should be discontinued before surgery is not known and is a source of some debate[22]. If radiographic contrast is needed, then newer isosmolar contrast agents may be less toxic.[23] In stable patients, cardiac surgery should be postponed in patients with contrast-induced ARF. Pharmacologic Interventions to Prevent PRD after Cardiac Surgery.[21] Pharmacologic interventions have been attempted with inconsistent results, and at this time, there are no known drugs that have demonstrated conclusively renal protection. The failure of these measures to prevent ARF after cardiac surgery may be related in part to a number of factors. First, the pathophysiology of ARF after CPB is more complex than originally considered and simple approaches to target single pathways are unlikely to succeed. Second, late pharmacologic intervention is likely to meet with failure. Third, patient populations that have been studied are often at low risk for renal dysfunction after CPB, thus potentially masking small beneficial effects of therapies. Last, most clinical trials enroll a small number of patients and are powered inadequately to detect small benefits. Most therapeutic trials inARF after CPB have been prevention studies in which treatment was initiated before the insult and in the majority of cases have shown no significant benefits. Diuretics may reduce the severity of ARF by preventing tubule obstruction and decreasing oxygen consumption.[24] In a double-blind, randomized, controlled trial, furosemide treatment was found not to be protective as the incidence of ARF was twice that of the dopamine or placebo group.[25] Similar negative results have been seen in other studies[24]. Mannitol has a variety of effects, including the production of an osmotic diuresis with a reduction of tubular obstruction, as well as the capability of scavenging free radicals. It is often added to the prime solution during CPB, with the thought that it may help to maintain urine output during the procedure, minimize tissue edema, and serve as a free radical scavenger.[25] An early study in children who underwent cardiac surgery demonstrated than prophylactic administration of mannitol $(0.5 \mathrm{~g} / \mathrm{kg}$ body wt $)$ was beneficial in the prevention of ARF.[26]Fisher et al.[27] demonstrated that mannitol added to the CPB prime solution was effective at maintaining urine output at varying doses. However, several other studies did not confirm these findings, and the potential role of mannitol remains unclear. In fact, Carcoana et al[28] showed an increased urinary excretion of B-2 microglobulin in patients who received mannitol and dopamine, suggestive of increased tubular injury in this group. Sirivella et al. randomly assigned 100 patients with postoperative oliguric or anuric renal failure to therapy with either intermittent doses of loop diuretics or a continuous infusion of mannitol, furosemide, and dopamine (2 $\mathrm{mg} / \mathrm{kg}$ per min).[29] Whereas $90 \%$ of patients who received the intermittent diuretic required dialysis, only $6.7 \%$ of the patients who received the continuous mannitol, furosemide, and dopamine infusion required dialysis. Furthermore, early therapy with this "cocktail" was associated with early restoration of 
renal function. Future studies are required before this approach can be broadly recommended. Drugs that Block Inflammation Inflammation is well documented to occur during CPB and has a prominent role in the pathogenesis of ARF and CPB.[30] It thus is an attractive therapeutic target. Pentoxifylline a phosphodiesterase inhibitor, blocks the activation of neutrophils by TNF- $\alpha$ and IL-1 and TNF- $\alpha$ release by inflammatory cells.[31] Pentoxifylline has been demonstrated to reduce cardiac dysfunction and TNF- $\alpha$ release in ischemia-reperfusion models[32]. However, pentoxifylline did not affect renal function in elderly patients who underwent cardiac surgery[33]. Dexamethasone also failed to protect against renal dysfunction after cardiac surgery.[14] A recent study examined the effect of blocking complement activation in patients who underwent CPB. A single-chain antibody specific for human C5 (pexelizumab) was found to block complement activation and postoperative myocardial injury. However, renal function was not an outcome measure of this pilot study.[34] N-acetylcysteine (N-AC) has been shown to block inflammation and oxidant stress in cardiac surgery patients and thus may hold promise as a simple, nontoxic protective measure.[35] However, $\mathrm{N}$-AC has not been used in a prospective clinical trial that examines renal outcomes. $\mathrm{N}$-AC has been studied most extensively in the prevention of radiocontrastinduced nephropathy. In this area, the utility of $\mathrm{N}-\mathrm{AC}$ has been questioned with the publication of a meta-analysis of 16 controlled studies that demonstrated no protective benefit.[36] Other Strategies. The sympathetic nervous system is activated during and after cardiac surgery and may lead to impairment of renal function through a hemodynamic mechanism. Clonidine (B2 agonist) has been used to attenuate these effects, withimprovement in hemodynamic stability during CPB[37] .In a study of 48 normal risk patients who underwent cardiac surgery, preoperative treatment with clonidine prevented the deterioration of renal function in this small trial, with creatinine clearances significantly higher in the clonidine-treated group $24 \mathrm{~h}$ after CPB.[38] Diltiazem has been used in clinical trials to prevent ARF after cardiothoracic surgery. Diltiazem has been shown to inhibit some of the inflammatory effects of CPB and is often used to prevent vasospasm of radial grafts.[39] Although diltiazem reduced urinary excretion of markers of tubule injury ( glutathione s-transferase and $\mathrm{N}$-acetyl-Bglucosaminidase), [40] its effectiveness in the prevention of renal dysfunction was inconsistent.[41] In patients who were at highest risk for PRD, prophylactic hemodialysis has been attempted10. In a single study, 44 patients with a baseline serum creatinine $>2.5 \mathrm{mg} / \mathrm{dl}$ were randomly assigned to either perioperative prophylactic dialysis or dialysis only when postoperative ARF that required the procedure was indicated (control). In the group that received prophylactic dialysis, mortality was 4.8 versus $30.4 \%$ in the control group. Furthermore, postoperative ARF that required dialysis was reduced from $34.8 \%$ in the control group to $4.8 \%$ in the intervention arm. These results will have to be repeated in other randomized, controlled studies before this invasive approach can be broadly recommended.

\section{Conclusions\&Recommendations:-}

From reviewing the literature and from our results in this study we concluded that:-

- $\square$ Safety and success of the offpump CABG surgery along with the new concerns about economics, together with advantages over CPB in avoidances of its recognized risks, added in resurrection of this technique in the past decade. $\bullet \square$ Analysis of postoperative results showed no correlation between findings in abdomio-pelvic U/S and postoperative renal impairment. $\bullet \square$ Cleveland Clinic Foundation Acute Renal Failure Scoring System does not take in consideration the various patho-physiological parameters of the CPB. $\bullet \square$ Throughout the literature there is a strong agreement that multiple risk factors (pre, intra, and postoperative) and multiple pathological and physiological factors interact together to affect the kidney functions. $\bullet \square$ In general, there is no single accurate investigation that can surely predict renal function outcome after cardiac surgery. $\bullet \square$ General Measures to prevent renal dysfunction after Cardiac Surgery should be utilized in all patients with special attention to those with preoperative renal dysfunction. $\bullet \square$ Regarding postoperative renal dysfunction and need for dialysis, results were in favor of the off-pump technique. $\bullet$ Renal function is better preserved in patients undergoing offpump CABG than those undergoing on-pump CABG. $\bullet$ CPB is associated with a higher risk for PRD, and this injury is associated further with substantial morbidity and mortality. $\bullet \square$ At present, no pharmacologic interventions have demonstrated conclusively efficacy in the prevention of renal dysfunction after cardiac surgery. $\bullet \square$ The pathogenesis of kidney injury during CPB is complex and involves hemodynamic, inflammatory, and other mechanisms that interact at a cellular level. $\bullet$ Ultimately, a successful therapy will utilize strategies that target these multiple pathways. This integrated strategy would target hemodynamic, inflammatory, and oxidative pathways and act at the points of proximal cellular injury. $\bullet$ CPB offers an attractive model to study these pathways, because the timing of the insult is known and potentially modifiable. 


\section{References:-}

1. Pitsis A, Mezilis N, Dardas P, Tsikaderis D, et al. Coronary Artery Bypass Grafting for Multi-Vessel Coronary Disease on the Beating Heart: Comparative Study of 500 Patients. Hellenic J cardiol 2002; 43: 26-31.

2. Ascione R, Lloyd CT, Gomes WJ, Caputo M, et al. Coronary revascularisation with or without cardiopulmonary bypass: evaluation of myocardial function in a prospective randomised study. Eur J Cardiothorac Surg 1999; 15: 685-690.

3. Zanardo G, Michieon P, Paccagnella A, Rosi P, et al. Acute renal failure in the patient undergoing cardiac operation: prevalence, mortality rate, and main risk factors. J Thorac Cardiovasc Surg 1994; 107: 1489-1495.

4. Hayashida N, Teshima H, Chihara $S$, Tomoeda $H$, et al. Does off pump coronary artery bypass grafting really preserve renal function? Circ J 2002; 66: 921-925

5. Boldt J, Berner T, Lehmann A, Suttner S, et al. Is kidney function altered by the duration of cardiopulmonary bypass? Ann Thorac Surg 2003; 75: 906-912.

6. Sheinbaum R, Ignacio $C$, Safi H, Estera A. Contemporary strategies to preserve renal function during cardiac and vascular surgery. Rev Cardiovasc Med 2003; 4: S21-S28

7. Byers J and Sladen R. Renal function and dysfunction. Curr Opin Anaesthesiol 2001; 14: 699-706.

8. Simmons $\mathrm{P}$ and Anderson R. Increased serum creatinine: A marker for adverse outcome before and after cardiac surgery. Crit Care Med 2002; 30: 1664-1665.

9. Durmaz I, Yagdi T, Calkavur T, Mahmudov R, et al. Prophylactic dialysis in patients with renal dysfunction undergoing on-pump coronary artery bypass surgery. Ann Thorac Surg 2003; 75: 859-864.

10. Lema G, Caessa R, Urzua J. Renal preservation in cardiac surgery. Curr Opin Anaesthesiol 1998; 11: 9-13.

11. Koutlas T, Elbeery J, Williams M, Moran J, et al. Myocardial revascularization in the elderly using beating heart coronary artery bypass surgery. Ann Thorac Surg 2000; 69: 1042-7.

12. Alserius T, Anderson RE, Hammar N, Nordqvist T, Ivert T. Elevated glycosylated haemoglobin (HbA1c) is a risk marker in coronary artery bypass surgery. Scand Cardiovasc J. 2008 Dec;42(6):392-8.

13. Khati NJ, Hill MC, Kimmel PL. The role of ultrasound in renal insufficiency: the essentials. Ultrasound Q. 2005 Dec; 21(4): 227-44.

14. Loef BG, Epema AH, Navis G, Ebels T, van Oeveren W, Henning RH. Off pump coronary revascularization attenuates transient renal damage compared with on-pump coronary revascularization. Chest. 2002 Apr;121(4):1190-4.

15. Pramodh K, Vani, Muralidhar K: Renal function following CABG: Onpump vs off pump Ind $\mathrm{J}$ Thorac Cardiovasc Surg, 2003;19: 169-173.

16. Wehlin L, Vedin J, Vaage J, Lundahl J Activation of complement and leukocyte receptors during on and off pump coronary artery bypass surgery European Journal of Cardio-thoracic Surgery 2004;25:35-42.

17. Limacher MC (1996) Coronary heart disease in women. Past gaps, present state and future promises. J Fla Med Assoc 1996; 83: 455-458.

18. Kaiser, Larry R.; Kron, Irving L.; Spray, Thomas L. Title: Mastery of Cardiothoracic Surgery, 2nd Edition Lippincott Williams \& Wilkins 2007.

19. Eagle KA, Guyton RA, Davidoff R, Edwards FH, Ewy GA, Gardner TJ, Hart JC, Herrmann HC, Hillis LD, Hutter AM Jr, Lytle BW, Marlow RA, Nugent WC, Orszulak TA; American College of Cardiology; American Heart Association. ACC/AHA 2004 guideline update for coronary artery bypass graft surgery: a report of the American College of Cardiology/American Heart Association Task Force on Practice Guidelines (Committee to Update the 1999 Guidelines for Coronary Artery Bypass Graft Surgery). Circulation. 2004 Oct 5;110(14):e340437.

20. Thakar CV, Arrigain S, Worley S, Yared J-P, Paganini EP: A clinical score to predict acute renal failure after cardiac surgery. J Am Soc Nephrol 2005;16: 162-168

21. Rosner MH, Okusa MD. Acute kidney injury associated with cardiac surgery. Clin J Am Soc Nephrol 2006; 1:19.

22. Lazar HL: The use of angiotensin-converting enzyme inhibitors in patients undergoing coronary artery bypass graft surgery. Vascul Pharmacol 2005; 42: 119-123.

23. Aspelin P, Aubry P, Fransson SG, Strasser R, Willenbrock R, Berg KJ; Nephrotoxicity in High-Risk Patients Study of Iso-Osmolar and Low- Osmolar Non-Ionic Contrast Media Study Investigators: Nephrotoxic effects in high-risk patients undergoing angiography. N Engl J Med 2003; 348: 491-499.

24. Jarnberg PO: Renal protection strategies in the perioperative period. Best Pract Res Clin Anaesthesiol 2004; 18: 645-660.

25. Lassnigg A, Donner E, Grubhofer G, et al. Lack of renoprotective effects of dopamine and furosemide during cardiac surgery. J Am Soc Nephrol 2000;11:97-104. 
26. Cooper JR, Giesecke NM: Hemodilution and priming solutions. In: Cardiopulmonary Bypass: Principles and Practice, edited by Gravlee GP, Davis RF, Kurusz M, Utley JR, Philadelphia, Lippincott Williams \& Wilkins, 2000, pp 186-196.

27. Rigden SP, Dillon MJ, Kind PR, de Leval M, Stark J, Barratt TM: The beneficial effect of mannitol on postoperative renal function in children undergoing cardiopulmonary bypass surgery. Clin Nephrol 1984; 21: 148-151.

28. Fisher AR, Jones P, Barlow P, Kennington S, Saville S, Farrimond J, Yacoub M: The influence of mannitol on renal function during and after open-heart surgery. Perfusion 1998; 13:181-186.

29. Carcoana OV, Mathew JP, Davis E, Byrne DW, Hayslett JP, Hines RL, Garwood S: Mannitol and dopamine in patients undergoing cardiopulmonary bypass: A randomized clinical trial. Anesth Analg 2003; 97: 1222-1229.

30. Sirivella S, Gielchinsky I, Parsonnet V: Mannitol, furosemide, and dopamine infusion in postoperative renal failure complicating cardiac surgery. Ann Thorac Surg 2000; 69: 501- 506.

31. Paparella D, Yau TM, Young E: Cardiopulmonary bypass induced inflammation: Pathophysiology and treatment. An update. Eur J Cardiothorac Surg 2002; 21: 232-244.

32. Cagli K, Ulas MM, Ozisik K, Kale A, Bakuy V, Emir M, Balci M, Topbas M, Sener E, Tasdemir O: The intraoperative effect of pentoxifylline on the inflammatory process and leukocytes in cardiac surgery patients undergoing cardiopulmonary bypass. Perfusion 2005; 20: 45-51.

33. Zhang M, Xu YJ, Saini HK, Turan B, Liu PP, Dhalla NS: Pentoxifylline attenuates cardiac dysfunction and reduces TNF-alpha level in the ischemic-reperfused heart. Am J Physiol Heart Circ Physiol. 2005; 289: 832839.

34. Boldt J, Brosch C, Piper SN, Suttner S, Lehmann A, Werling C: Influence of prophylactic use of pentoxifylline on postoperative organ function in elderly cardiac surgery patients. Crit Care Med 2001; 29: 952-958.

35. Shernan SK, Fitch JC, Nussmeier NA, Chen JC, Rollins SA, Mojcik CF, Malloy KJ, Todaro TG, Filloon T, Boyce SW, Gangahar DM, Goldberg M, Saidman LJ, Mangano DT; Pexelizumab Study Investigators: Impact of pexelizumab, an anti-C5 complement antibody, on total mortality and adverse cardiovascular outcomes in cardiac surgical patients undergoing cardiopulmonary bypass. Ann Thorac Surg 2004;

36. 77: 942-949.

37. Sucu N, Cinel I, Unlu A, Aytacoglu B, Tamer L, Kocak Z, Karaca K, Gul A, Dikmengil M, Atik U, Oral U: Nacetylcysteine for preventing pumpinduced oxidoinflammatory response during cardiopulmonary bypass. Surg Today 2004; 34: 237-242.

38. Kshirsagar AV, Poole C, Mottl A, Shoham D, Franceschini N, Tudor G, Agrawal M, Denu-Ciocca C, Magnus Ohman E, Finn WF: Nacetylcysteine for the prevention of radiocontrast induced nephropathy: A meta-analysis of prospective controlled trials. J Am Soc Nephrol 2004; 15: 761-769.

39. Myles PS, Hunt JO, Holdgaard HO, McRae R, Buckland MR, Moloney J, Hall J, Bujor MA, Esmore DS, Davis BB, Morgan DJ: Clonidine and cardiac surgery: Haemodynamic and metabolic effects, myocardial ischaemia and recovery. Anaesth Intensive Care 1999; 27: 137-147.

40. Kulka PJ, Tryba M, Zenz M: Preoperative alpha2-adrenergic receptor agonists prevent the deterioration of renal function after cardiac surgery: Results of a randomized, controlled trial. Crit Care Med 1996; 24: 947-952.

41. Fansa I, Gol M, Nisanoglu V, Yavas S, Iscan Z, Tasdemir O: Does diltiazem inhibit the inflammatory response in cardiopulmonary bypass? Med Sci Monit 2003; 9: I30-I36.

42. Bergman AS, Odar-Cederlof I, Westman L, Bjellerup P, Hoglund P,Ohqvist G: Diltiazem infusion for renal protection in cardiac surgical patients with preexisting renal dysfunction. J Cardiothorac Vasc Anesth 2002; 16: 294-299.

43. Piper SN, Kumle B, Maleck WH, et al. Diltiazem may preserve renal tubular integrity after cardiac surgery. Can J Anaesth 2003;50:285- 292. 